\section{WEB WATCH}

Free associations

- The Genetic Association Database:

http://www.grc.nia.nih.gov/ branches/rrb/dna/ association

Association studies have finally found a home of their own. The steadily increasing number of these studies which report a link between one or more genetic polymorphisms and specific human disorders - is filling the literature databases. So, researchers have for some time needed a single, comprehensive repository of all the results that have been published so far. Such a resource has now become available thanks to efforts headed by Kevin Becker at the National Institute on

Aging $/ \mathrm{NIH}$, who has compiled a freely accessible web site the Genetic Association

Database (GAD) - that catalogues the results of over 700 association studies. The site is still under development and will grow both in content and utility. Nevertheless, its features are clear to see. The studies can be queried according to disease and phenotypic information, as well as according to various aspects of a study's design, such as study sample size or the statistical significance of the association. Links are available, through PubMed, to the original publication, and comments that could be potentially useful to other investigators can also be posted for each study. Importantly, the database also includes studies in which no genetic association was found.

With 75-100 association studies published each week, GAD should attract a lot of traffic. Anyone can submit the results of an association study to the site, provided that permission is obtained from the curator. The future of the database - the only one of its kind - could be made even rosier if Becker realizes his ambition to enrich the site by integrating it with geneexpression and $\mathrm{NCB}$ databases.

\section{Cutting out a pattern}

Protein degradation is a key regulator of the cell cycle, inflammation and transcription - but little is known

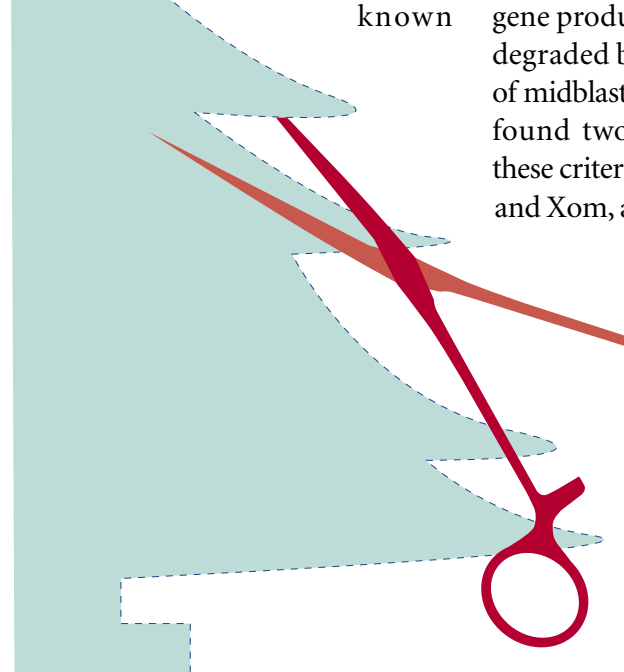

about its role in development. Zhu and Kirschner now report in Developmental Cell the identification of Xom - a developmental gene that is regulated by proteolysis.

In a screen, in Xenopus, to identify gene products that are differentially egraded before and after the onset astula transition, the author two proteins that matched criteria - an unknown protein homeobox transcription factor. Xom is stable during e a r ly gastrula tion but is subsequently degraded through the ubiquitin-proteasome pathway.

Xom has two socalled PEST domains (proline, aspartate and glutamate,

serine, or threonine-rich regions), one of which turned out to be required for Xom degradation. Interestingly, this so-called 'Xom destruction motif' (XDM) resembles the glycogen synthase kinase 3 (GSK3) consensus phosphorylation site, which is conserved in the known substrates of GSK3dependent proteolysis, such as $\beta$-catenin.

In the XDM, the authors found two phosphorylation sites at Ser 140 and Ser144. In an in vitro assay, an exogenous peptide of the phosphorylated XDM blocked Xom degradation, paradoxically so did the unphosphorylated peptide. However, phosphorylation of XDM seems to be important for Xom degradation because the same peptide, when it contains serine-to-alanine mutations at positions 140 and 144, cannot block Xom degradation in vitro, implying that the embryonic extract used in these assays contained a kinase activity, although this turned out not to be GSK3.

Using in vitro binding assays, Zhu and Kirschner next found that the E3 ubiquitin ligase Skp1Cullin-F-box complex (SCF),

\title{
A clear suspect
}

Approximately $0.05 \%$ of the Western population suffers from systemic lupus erythematosus (SLE) - a complex autoimmune disease. Although several susceptibility loci for SLE have been identified, the nature of the genes and mutations that underlie this disease have remained unknown. Now, Prokunina et al. report in Nature Genetics the association of the programmed cell death gene 1 (PDCD1) with SLE. Importantly, they also propose how a particular sequence variant of $P D C D 1$ might contribute to the disease's aetiology.

In a previous study of a Nordic population, the authors identified a susceptibility locus for SLE on chromosome 2 . One gene in this region stood out as a potential candidate, $P D C D 1$. This is because $P D C D 1$ encodes an immunoreceptor that belongs to the immunoglobulin family and that is known to regulate peripheral self-tolerance in $\mathrm{T}$ and B cells. Moreover, $P d c d 1^{-1-}$ mice suffer from SLE-like symptoms.

By sequencing PDCD1 in five healthy unrelated individuals and in five SLE sufferers from the Nordic population, the authors discovered seven SNPs in this gene, three of which constituted a disease-associated haplotype that could account for all of the LOD score seen in the original population sample. These SNPs were then genotyped in five sets of families with different ethnic origins. The results were clear only one SNP, which lies in an enhancer-like region in intron 4 of PDCD1, consistently associated with SLE. This region of intron 4 contains binding sites for transcription factors that are known to be involved in haematopoietic differentiation and in inflammation. In particular, the SNP disrupts a putative binding site for RUNX1, which is inactivated in translocations that lead to acute myeloid leukaemia. Using an electrophoretic mobility shift assay, the authors confirmed that RUNX1 indeed binds to this sequence and that this binding is abolished by the sequence change that is associated with the SNP.

The authors propose that RUNX1 binding to the wild-type 